\title{
The Combined Effects of Mesenchymal Stem Cell Conditioned Media and Low-Level Laser on Stereological and Biomechanical Parameter in Hypothyroidism Rat Model
}

\author{
Niloofar Sefati' ${ }^{1}$ Hojjat-Allah Abbaszadeh2,1* , Fatemeh Fadaei Fathabady,1, Mohammad-Amin Abdollahifar ${ }^{1}$, \\ Maryam-Sadat Khoramgah ${ }^{2}$, Shahram Darabi ${ }^{4}$, Abdollah Amini ${ }^{1}$, Foozhan Tahmasebinia ${ }^{5}$, Mohsen Norouzian ${ }^{3,1 *}$ \\ ${ }^{1}$ Department of Biology and Anatomical Sciences, School of Medicine, Shahid Beheshti University of Medical Sciences, \\ Tehran, Iran \\ ${ }^{2}$ Hearing Disorders Research Center, Loghman Hakim Medical Center, Shahid Beheshti University of Medical Sciences, \\ Tehran, Iran \\ ${ }^{3}$ Laser Application in Medical Sciences Research Center, Shahid Beheshti University of Medical Sciences, Tehran, Iran \\ ${ }^{4}$ Cellular and Molecular Research Center, Qazvin University of Medical Science, Qazvin, Iran \\ ${ }^{5}$ Department of Biological Sciences, Institute for Advanced Studies in Basic Sciences (IASBS), Zanjan, Iran
}

\section{*Correspondence to \\ Hojiat-Allah Abbaszadeh, Assistant \\ Professor, Hearing Disorders Research \\ Center, Shahid Beheshti University of Medical Sciences, Tehran, Iran. \\ Email:dr.abbaszadeh@sbmu.ac.ir; Mohsen Norouzian, Associate \\ Professor, Department of Biology and Anatomical Sciences, School of Medicine, Shahid Beheshti University of Medical Sciences, Tehran, Iran. Tel: +982123872555 \\ Email: norozian93@gmail.com}

Published online 17 September 2018

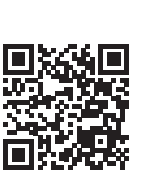

\begin{abstract}
Introduction: Many studies have shown the positive effect of laser radiation and application of the mesenchymal stem cells (MSCs) and their secretion in stimulating bone regeneration. The aim of this study was determining effects of MSC conditioned media (CM) and low-level laser (LLL) on healing bone defects in the hypothyroid male rat.

Methods: We assigned 30 male Wistar rats randomly to 3 groups: control, hypothyroidism, CM+LLL. Four weeks after surgery, the right tibia was removed. Biomechanical examination and histological examinations were performed immediately.

Results: Our results showed significant increase in bending stiffness $(116.09 \pm 18.49)$, maximum force $(65.41 \pm 8.16)$, stress high load $(23.30 \pm 7.14)$, energy absorption $(34.57 \pm 4.10)$, trabecular bone volume $(1.34 \pm 0.38)$ and the number of osteocyte, osteoblast, and osteoclast $(12.77 \pm 0.54$, $6.19 \pm 0.80,1.12 \pm 0.16$ respectively) in osteotomy site in the $L L L+C M$ group compared to the hypothyroidism group $(P<0.05)$.

Conclusion: The results indicated that using the LLL + CM may improve fracture regeneration and it may hasten bone healing in the hypothyroid rat.

Keywords: Hypothyroidism; Low-level laser; Conditioned Media
\end{abstract}

\section{Introduction}

The hormones secreted from the thyroid gland play very important role in the evolution of various body systems, including nervous and skeletal system, as well as the differentiation of different cells. ${ }^{1,2}$ Hypothyroidism, also called low thyroid state, is a disorder of the endocrine system where the thyroid gland fails to produce enough thyroid hormone. It can cause a number of symptoms, such as cold intolerance, fatigue, constipation and depressed mood. ${ }^{3-5}$ Untreated hypothyroidism during pregnancy can lead to growth retardation and intellectual difficulties. The worse type of congenital hypothyroidism is called cretinism which is a cause of severe mental retardation and growth delays. ${ }^{6}$ Reduced circulating thyroid hormones or lack of active receptors gives rise to a variety of disorders in the metabolism of the body.
In the musculoskeletal system, hypothyroidism disrupts bone formation and reabsorption and impairs bone regeneration. It leads to up to 3 times increase in the risk of fractures. ${ }^{7-9}$ The treatment of hypothyroidism is hormone replacement which usually should be continued throughout the whole life. ${ }^{10,11}$ On the other hand, treatment with thyroid hormones can increase the risk of osteoporosis and fracture risk. One of the modalities that are used for inducing the bone repair is laser therapy. ${ }^{12,13}$ The laser radiation can be absorbed by the mitochondrial chains, which increases the production of ATP, production of oxygen, protein synthesis and ultimately increased mitosis and cell proliferation by inducing osteogenic gene expression. In addition laser can elevate osteoblast activity and bone formation. ${ }^{14-16}$ Other proposed therapeutic methods are the application of growth factors derived

Please cite this article as follows: Sefati N, Abbaszadeh HA, Fadaei Fathabady F, et al. The combined effects of mesenchymal stem cell conditioned media and low-level laser on stereological and biomechanical parameter in hypothyroidism rat model. I Lasers Med Sci. 2018;9(4):243-248. doi:10.15171/jlms.2018.44. 
from various sources. Mesenchymal stem cells (MSCs) are multipotent cells with abundance. ${ }^{14}$ The growth factors secreted from MSCs accumulate in a conditioned culture media during tissue culture, which can increase tissue repair and angiogenesis and reduce apoptosis., ${ }^{7,16}$ These cytokines have considerable effect on the processes of survival, migration, proliferation, and differentiation of cells, especially angiogenesis, osteogenesis, bone remodeling and bone fracture repair. ${ }^{17,18}$ Considering the effect of hypothyroidism on bone formation and undesirable side effects of conventional treatments, we investigated the effect of LLL and CM derived from MSC in the treatment of bone fractures of hypothyroid rats.

\section{Methods}

Animal

In this study, we used 30 Wistar rats (200-220 g). They were kept at the research site for two weeks. During the study, the rats had free access to water and standard food. Animal conditions were controlled at a temperature of about $22^{\circ} \mathrm{C}$ and a 12 -hour brightness cycle.

Extraction of Mesenchymal Stem Cell

After sacrificing the rats, in a sterile condition, femur and tibia bones on each side were extracted. After removing muscles and tissues, the epiphyses of each side of femur and tibia bone were cut. The bone marrow was extracted using a $5 \mathrm{cc}$ syringe containing $4 \mathrm{ml}$ of DMEM (Gibco, USA). The cells derived from the bone marrow were put inside a culture flask in a DMEM and antibiotic mixture $(100 \mathrm{u} / \mathrm{ml}$ Penicillin $\mathrm{G}$ and $100 \mathrm{mg} / \mathrm{mL}$ Streptomycin, both from Gibco, USA). Supplementation was done with $10 \%$ FBS. The cells of the fourth passage of culture were used for this study. ${ }^{19,20}$

\section{Determination of Surface Markers of MSC Using Flow Cytometry \\ In this study, MSCs were evaluated for the presence of CD34, CD44, CD45 and CD90 using flow cytometry. After preparing the appropriate suspension, the cells coloured with fluorescence material or labeled with conjugated antibodies with a fluorochrome.}

Preparation of Mesenchymal Stem Cells-Conditioned Media

In order to obtain the conditioned culture media of bone marrow stem cells (MSCs-CM), we seeded the fourth passage of the MSC at a density of 10000 cells $/ \mathrm{cm}^{2}$. At $80 \%$ confluence, the cells were washed 3 times with phosphate buffer (PBS). We replaced the media with serum-free DMEM (Sigma, USA). After 48 hours, cells were incubated and the media were stored at $-80^{\circ} \mathrm{C}$ until use. Using lyophilized-drying (Christ Alpha1-2 LD Plus, Germany), according to the manufacturer's instructions the media was concentrated 20 fold before in vivo application. $^{7}$
Induction of Hypothyroidism and Surgical Procedure The rats daily received $4 \mathrm{mg}$ powder methimazole (Sigma, USA) for 4 weeks. After induction hypothyroidism the rats were anesthetized by intramuscular injection of ketamine and diazepam. After cutting into the skin and pulling the muscles away, a fine-toothed circular micro saw was inserted and a $1.5 \mathrm{~mm}$ hole in the tibia bone of the 2 sides was created with a minor lesion at the midpoint of the tibia. The muscle was sewn by catgut 04 absorbing suture and the skin was sewn by the reverse twist of the nylon thread. The animals received intramuscular ceftriaxone as a prophylactic antibiotic. ${ }^{7}$

\section{Research Groups}

After bone defect, rats were randomly assigned into 5 groups:

Control group: Rats were developed without any bone defect and without methimazole and then were kept for one month without any intervention. Hypothyroid group: hypothyroidism was induced by methimazole and the bone defect was mentioned before and then the rats were kept for 1 month without any other intervention.

$L L L+C M$ group: After inducing hypothyroidism and performing a bilateral osteotomy, the rat simultaneously received the LLL (Infrared [GaAlAs] lasers with a wavelength of $890 \mathrm{~nm}$ and a frequency of $80 \mathrm{~Hz}$ for 20 minutes for each point at a dose of $1.5 \mathrm{~J} / \mathrm{cm}^{2}$. Up to 3 points including the site of the bone defect, $0.5 \mathrm{~cm}$ below and 0.5 $\mathrm{cm}$ above the defect were treated with the laser) and the $\mathrm{CM}$ in the above-mentioned methods. Four weeks after the surgery, the rat was sacrificed and their right and left tibia bone was sampled. The right tibia was undergone biomechanical experiments and left tibia was used for histological studies. Statistical analysis was performed on the obtained data.

\section{Biomechanical Test (3-Point Test)}

The 2 ends of the bone were placed on the 2 edges of the support holders of the material strength test (Zwick, Germany). The distance between the 2 supporting points was $20 \mathrm{~mm}$. All samples were placed in a similar orientation. ${ }^{11}$

\section{Hematoxylin-Eosin Staining}

The bone was transferred to a $10 \%$ buffered formalin solution and then was placed in a solution of $10 \%$ nitric acid for 1 week to decalcify. Then, it stained with hematoxylin-eosin methods.

\section{Stereology Study}

Volume Estimation

Cavalri technique was used to determine bone volume. To this, the order of 10 sections of a sample was selected randomly. Then, a multipurpose test system with 100 points was used to calculate the bone volume, so that the 
points of the grid were randomly placed on the sections of the tissue and counting the number of points, the total volume of the bones using the formula: $V=\Sigma p \times a / p \times t$. In this formula " $\Sigma \mathrm{p}$ " is the number of points of the test system placed on the tissue, " $\mathrm{a} / \mathrm{p}$ " is the area of each point and " $\mathrm{t}$ " is the distance between the sampled sections. ${ }^{21}$

\section{Number Estimation}

We used optical disector to calculate the number of bone cells. To calculate the number of bone cells due to their different sizes, 10 micron sections were used, so that 10 sections were randomly selected. Then, the entire field of view of each section was examined using Nikon, Japan (E200) microscope. According to the counting method on a special counting frame, cells that their cores were counted in the frame or on the lines were selected, those on the forbidden lines were not accepted, so were not counted. Then, the numerical value of the types of bone cells was calculated according to the following equation:

$\mathrm{N}($ total $)=\mathrm{Nv} \times \mathrm{V}($ ref $)$

Then the density was multiplied by total bone volume to obtain the absolute number of cells.In this formula " $\Sigma Q$ " is the number of the nuclei coming into focus and counted, " $\Sigma \mathrm{P}$ " is the total number of the counting frames in all fields, " $a / f$ " is the area per frame, " $h$ " is the height of the disector, " $\mathrm{t}$ " is the real section thickness, and "BA" is the block advance of the microtome. ${ }^{22}$

\section{Statistical Analysis Method}

The determination of the normal distribution of data was done by Kruskal-Wallis test and testing the statistical difference between the groups studied in different parts of the investigation. One-way analysis of variance (ANOVA) and LSD were used in the case of normal data distribution, and Kruskal-Wallis test and Mann-Whitney test were applied in the case of failure and natural distribution of data. The significant level of $P<0.05$ was considered for ANOVA, LSD and $P<0.01$ for Mann-Whitney test.

\section{Results}

Confirmation of Mesenchymal Stem Cell Properties

Since these cells contained some nonadhesive cell in primitive cultures, they were removed by cell passages, and most of the impurities were removed in passage 4 . Figure 1 shows the results of the flow cytometry technique confirming the markers of MSC. The cells expressed high percentage mesenchymal surface markers (CD44, CD90), in which the markers of hematopoietic cells (CD34 and CD45) did not express.

\section{Thyroid Hormones Level}

The results indicated that the induction of hypothyroidism by methimazole reduced $\mathrm{T} 3$ and T4 levels in the peripheral blood (Table 1).

Three-Point Biomechanical Test Analysis Analysis of ANOVA was performed for evaluating the biomechanical data in different groups and the results are shown in Table 2. We observed a significant difference between the hypothyroidism and the control group in terms of the bending stiffness $(P<0.01)$. A significant difference was seen between the hypothyroid group and
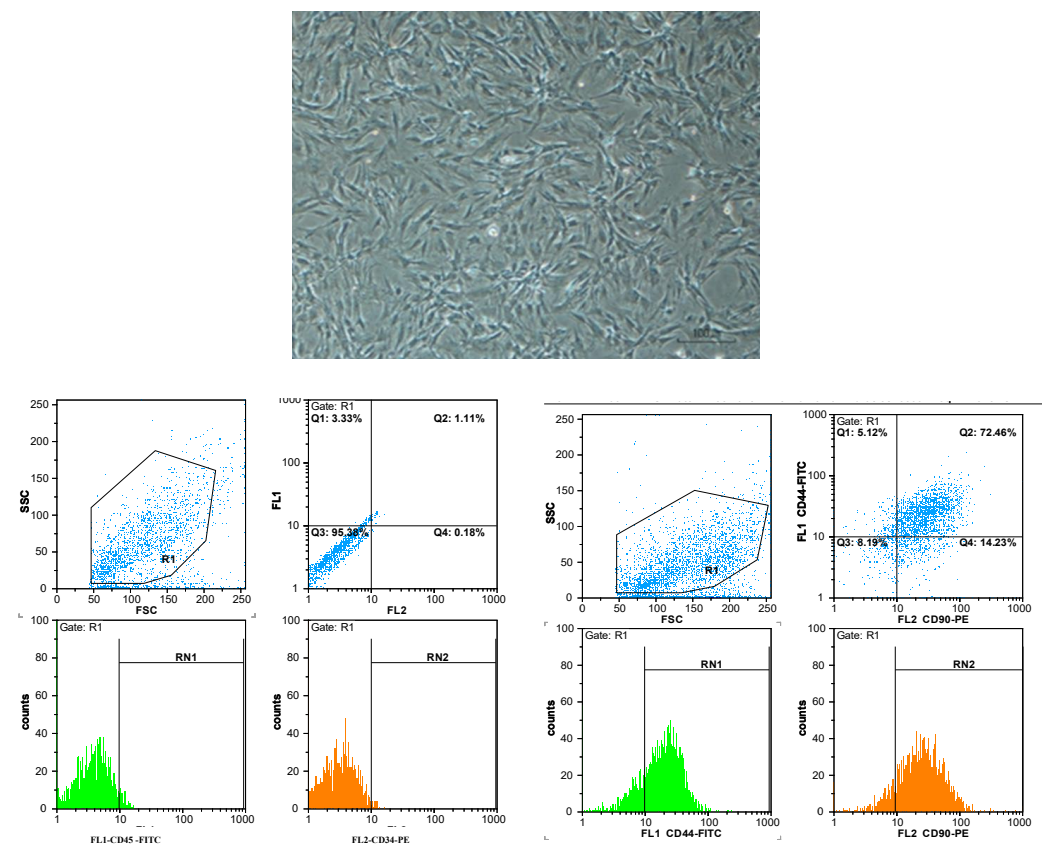

Figure 1. Microscopic image of mesenchymal stem cells in the fourth passage and results of flowcytometry indicate that these cells express the MSC marker, including CD44 and CD90 with high percentages, while they do not express the parameters of the hematopoietic cells $(\mathrm{CD} 34,45)$ 
Table 1. Thyroid Hormones Levels Before and After Hypothyroidism $(P<0.05)$

\begin{tabular}{lll}
\hline Thyroid Hormone & Preinduction & Postinduction \\
\hline $\mathrm{T} 3$ & $94.29 \pm 1.14 \mathrm{ng} / \mathrm{dL}$ & $22.44 \pm 2.81 \mathrm{ng} / \mathrm{dL}$ \\
$\mathrm{T} 4$ & $4.02 \pm 1.56 \mu \mathrm{g} / \mathrm{dL}$ & $2.01 \pm 2.16 \mu \mathrm{g} / \mathrm{dL}$ \\
\hline
\end{tabular}

Table 2. Results of Biomechanical Test Analysis of 3 Points by the Groups Studied $(P<0.01)$.

\begin{tabular}{lccc}
\hline & \multicolumn{3}{c}{ Groups } \\
\cline { 2 - 4 } & Control & Hypothyroid & LLL+CM \\
\hline Bending stiffness & $104.12 \pm 54.41$ & $34.86 \pm 2.23$ & $116.09 \pm 18.49$ \\
Maximum force & $48.22 \pm 6.23$ & $16.74 \pm 2.81$ & $65.41 \pm 8.16$ \\
Stress high load & $17.32 \pm 1.59$ & $8.14 \pm 4.22$ & $23.30 \pm 7.14$ \\
Energy absorption & $28.21 \pm 5.32$ & $11.09 \pm 3.32$ & $34.57 \pm 4.10$ \\
\hline
\end{tabular}

the LLL + CM in stress high load $(P<0.01)$. The difference between the hypothyroidism and other groups was also significant in maximum force $(P<0.01)$. A difference was observed between the hypothyroid and the control group in terms of the stress high load $(P<0.05)$. There was no significant difference between the treatment groups in terms of stress high load but a statistically significant difference existed in terms of energy absorption between the hypothyroid and control groups, LLL $+\mathrm{CM}(P<0.01)$.

Analysis of Stereological Parameters

The results of comparing different groups are shown in Table 3. There was a significant difference in the volume of bone marrow between the LLL+CM and the control group $(P<0.05)$. No meaningful difference observed in terms of the cortical tissue volume but there was a significant difference in the volume of trabecular tissue between the hypothyroid and the control group $(P<0.05)$. The difference between the hypothyroid and the LLL + $\mathrm{CM}$ group for trabecular tissue volume was significant $(P<0.05)$ (Figure 2).

The results for the number of bone cells are shown in Table 4. There was a significant difference between the number of osteocytes between hypothyroid and control groups $(P<0.05)$. There was also a significant difference in the number of osteocytes between hypothyroid and LLL $+\mathrm{CM}(P<0.01)$. We found also a significant difference

Table 3. Data on Bone Density $\left(10^{6}\right)$ Divided Into Different Groups $(P<0.05)$

\begin{tabular}{llll}
\hline & BMV & CBV & TBV \\
\hline Groups & & & \\
Control & $0.64 \pm 0.35$ & $2.54 \pm 2.49$ & $1.23+0.94$ \\
Hypothyroid & $0.76 \pm 0.65$ & $1.96 \pm 0.34$ & $0.42+0.21$ \\
LLL+CM & $0.49 \pm 0.23$ & $2.22 \pm 0.16$ & $1.34 \pm 0.38$ \\
\hline
\end{tabular}

Abbreviations: BMV, bone marrow volume; CBV, cortical bone volume; TBV, trabecular bone volume.
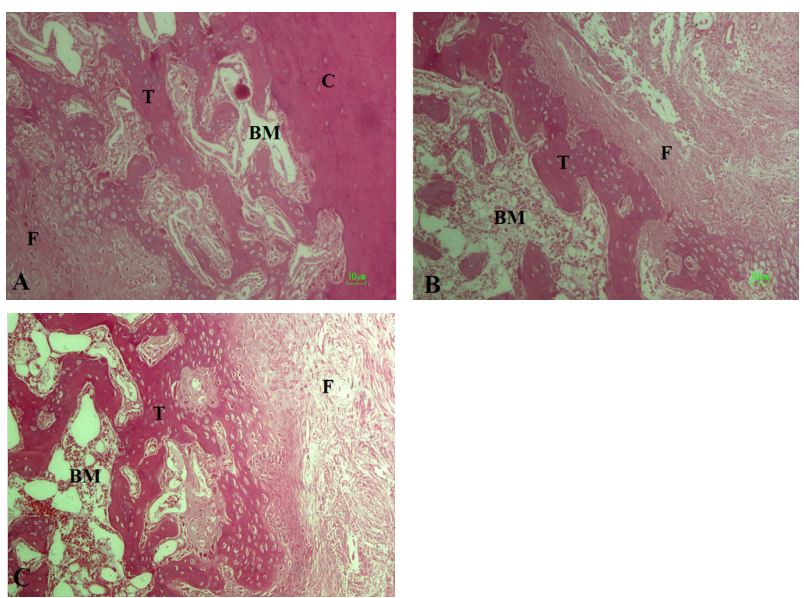

Figure 2. Micrograph of the Bone Defect Stained With H\& E. (A) Control; (B) Hypothyroidism; (C) CM+LLL. Trabecular bone (T), bone marrow $(\mathrm{BM})$, fibrous tissue $(\mathrm{F})$, and cortical bone.

in the number of osteoblasts between the hypothyroid and the control group $(P<0.05)$. The difference in the number of osteoblasts was statistically significant between hypothyroid and LLL + CM group $(P<0.01)$. A significant difference was found in the number of osteoclasts between the hypothyroid and the control group $(P<0.05)$. Furthermore, there was a significant difference between the number of osteoclasts between the hypothyroid and the LLL + CM group $(P<0.05)$.

\section{Discussion}

Decrease in circulation of the thyroid hormones or their receptors causes a variety of disorders in the metabolism of the body including disruption of normal bone formation and repair. Generally, hypothyroidism reduces the activity of osteoblasts, bone resorption impairment (due to decrease in the number and function of osteoclasts), and interferes in bone regeneration, delays bone formation and diminishes the bone growth. ${ }^{23}$ Studies have shown that prolonged use of levothyroxine could also reduce bone density and increase the risk of osteoporosis, which results delayed fracture repair in patients with hypothyroidism and elevates risk of fracture. ${ }^{24}$ The results of our study confirmed the previous reports saying that hypothyroidism is attributed with the decrease in the number of bone cells and also delays the repair of fractures following the reduction of the trabecular bone formation. ${ }^{3,4}$ Regarding these effects on the skeletal system,

Table 4. Data on the Number of Bone Marrow $\left(10^{3}\right)$ in Different Groups $(P<0.05)$

\begin{tabular}{llll}
\hline & Osteocyte & Osteoblast & Osteoclast \\
\hline Groups & & & \\
Control & $12.44 \pm 2.67$ & $5.87 \pm 4.23$ & $1.46 \pm 0.86$ \\
Hypothyroid & $5.01 \pm 0.54$ & $2.68 \pm 1.66$ & $0.49 \pm 0.26$ \\
LLL+CM & $12.77 \pm 0.54$ & $6.19 \pm 0.80$ & $1.12 \pm 0.16$ \\
\hline
\end{tabular}


treating bone defects caused by trauma, tumor removal, and other insults in a hypothyroid patient is a major challenge. ${ }^{6}$ It necessitates studying various therapeutic methods, including the use of MSCs or their secretions as an optimum culture media and LLL in order to find an appropriate and efficient remedy for treatment of bone injuries in hypothyroidism. The results of our study showed that CM+LLL improve histological parameters of repaired bone following a bone lesion. It leads to an increase in the volume of trabecular bone and improved biomechanical parameters such as maximum force and elasticity. A study by Hao et al showed that optimum culture media contains factors such as cytokines and chemokines that contribute to angiogenesis and repair of bone fractures and can increase the migration of other cells to the site of injury. ${ }^{25}$ The results of this study also showed that LLL improves biomechanical parameters such as stress high load. It also enhances histological parameters and brings about an increased volume of trabecular bone in rat. Several reports are available about the beneficial effects of low-energy laser radiation. Studies have shown that LLL radiation can have stimulatory effects on bone lesions. Laser irradiation energy can be absorbed by the mitochondrial chains, which in turn increases mitosis and proliferation of the cell. ${ }^{26}$ Osteogenic effects of pulsed LLL have also been reported on bone proliferation both in vitro and in vivo. ${ }^{27}$ Garavello et al compared the effects of pulsed ultrasound and LLL on bone repair following partial tibial bone defects. Histologic studies showed that in both laser and ultrasound groups the number of osteoblasts were more than control group. They concluded that ultrasound accelerates bone resorption through absorption and LLL improves recovery through bone formation. ${ }^{28}$ The results of our study showed that combination therapy with CM derived from MSC and LLL improves biomechanical parameters including stress high load and energy absorption. It also improves histological parameters in rats. Our results confirmed the previous studies showing that laser with a density of 1.5 $\mathrm{J} / \mathrm{cm}^{2}$ can increase the biomechanical bone parameters. ${ }^{7}$ It can elevate the maximum force, maximum stress, and energy absorption in comparison to the nontreatment group. Our histologic results confirmed previous studies $^{12}$ and LLL increased the trabecula bone density. We demonstrated a significant difference in the volume of bone in the LLL + CM group. These results can indicate that the bone formation effects of LLL are enhanced by the CM. Simultaneous application of CM and LLL can improve biomechanical and histological parameters even further compared to other groups. The simultaneous use of CM and LLL can also increase the bone cells count (osteoblasts, osteoclasts, and osteocytes) and the subsequent formation of the new bone in the defect site. Other studies confirmed that using bone scaffolds for delivery of CM can elevate its effect on fracture repair. ${ }^{29,30}$

\section{Conclusion}

Our results showed that treatment with $\mathrm{CM}$ along with simultaneous LLL can help in the quick repair of bone fractures. Moreover, application of LLL and CM together significantly increase the bearing potentials of stress high load, maximum force, stress high load as well as trabecular tissue volume and number of osteoblasts and osteoblasts.

\section{Ethical Considerations}

The study was approved by Shahid Beheshti University of Medical Sciences (Ethics No. IR.SBMU.SM.REC.1394.31).

\section{Conflict of Interests}

The authors declare no conflict of interest.

\section{Acknowledgments}

We are grateful for the support provided by Laser Application in Medical Sciences Research Center, Shahid Beheshti University of Medical Sciences, Tehran, Iran.

\section{References}

1. Wolf M, Ingbar SH, Moses AC. Thyroid hormone and growth hormone interact to regulate insulin-like growth factor-I messenger ribonucleic acid and circulating levels in the rat. Endocrinology. 1989;125(6):2905-29014. doi:10.1210/endo-125-6-2905

2. Garber JR, Cobin RH, Gharib H, et al. Clinical practice guidelines for hypothyroidism in adults. Thyroid. 2012;22(12):1200-1235.

3. Karimifar M, Esmaili F, Salari A, Kachuei A, Faragzadegan Z, Karimifar M. Effects of levothyroxine and thyroid stimulating hormone on bone loss in patients with primary hypothyroidism. J Res Pharm Pract. 2014;3(3):83-87. doi:10.4103/2279-042X.141099

4. Klubo-Gwiezdzinska J, Wartofsky L. Thyroid emergencies. Med Clin North Am. 2012;96(2):385-403.

5. Kindblom J, Gothe S, Forrest D, Tornell J, Vennstrom B, Ohlsson C. GH substitution reverses the growth phenotype but not the defective ossification in thyroid hormone receptor alpha 1-/-beta-/-mice. J Endocrinol. 2001;171(1):15-22.

6. Chakera AJ, Pearce SH, Vaidya B. Treatment for primary hypothyroidism: current approaches and future possibilities. Drug Des Devel Ther. 2012;6: 1-11. doi:10.2147/DDDT.S12894

7. Freidouni M, Nejati H, Salimi M, et al. Evaluating glucocorticoid administration on biomechanical properties of rats' tibial diaphysis. Iran Red Crescent Med J. 2015;17(3): e19389.

8. Son Y, Cheong YK, Kim NH, Chung HT, Kang DG, Pae HO. Mitogen-activated protein kinases and reactive oxygen species: how can ROS activate MAPK pathways? J Signal Transduct. 2011;2011:792639. doi:10.1155/2011/792639

9. Fernandes KR, Ribeiro DA, Rodrigues NC, et al. Effects of low-level laser therapy on the expression of osteogenic genes related in the initial stages of bone defects in rats. J Biomed Opt. 2013;18(3):038002. doi:10.1117/1. 
JBO.18.3.038002

10. Freitas FR, Capelo LP, O'Shea PJ, et al. The thyroid hormone receptor $\beta S$ pecific agonist GC1 selectively affects the bone development of hypothyroid rats. J Bone Miner Res. 2005;20(2):294-304.doi:10.1590/2359-3997000000027

11. Firouzi A, Fadaei Fathabadi F, Norozian M, Amini A, Abdollahifar MA, Noruzian M. The combined effects of levothyroxine and low level laser therapy on wound healing in hypothyroidism male rat model. J Lasers Med Sci. 2018;9(1):7-10. doi:10.15171/jlms.2018.02

12. Bolognani L, Volpi N. Low power laser enzymology: reactivation of myosin ATPase by $\mathrm{GaAs}$ an $\mathrm{He} / \mathrm{Ne}$ lasers. In: Passarella S, Quagliariello E, eds. Basic and Applied Research in Photobiology and Photomedicine. Bari, Italy: University of Bari; 1991:315-338.

13. Gao X, Xing D. Molecular mechanisms of cell proliferation induced by low power laser irradiation. J Biomed Sci. 2009;16:4. doi:10.1186/1423-0127-16-4

14. Mohsenifar Z, Fridoni $M$, Ghatrehsamani $M$, et al. Evaluation of the effects of pulsed wave LLLT on tibial diaphysis in two rat models of experimental osteoporosis, as examined by stereological and real-time PCR gene expression analyses. Lasers Med Sci. 2016; 31(4):721-732. doi:10.1007/s10103-016-1916-9

15. Mostafavinia A, Razavi S, Abdollahifar M, Amini A, Pouriran R, Bayat M. Evaluation of the effects of photobiomodulation on bone healing in healthy and streptozotocin-induced diabetes in rats. Photomed Laser Surg. 2017;35(10):537-545. doi:10.1089/pho.2016.4224.

16. Salgado AJ, Reis RL, Sousa NJ, Gimble JM. Adipose tissue derived stem cells secretome: soluble factors and their roles in regenerative medicine. Curr Stem Cell Res Ther. 2010;5(2):103-10.

17. Peyvandi AA, Abbaszadeh HA, Roozbahany NA, et al. Deferoxamine promotes mesenchymal stem cell homing in noise-induced injured cochlea through PI3K/ AKT pathway. Cell Proliferation J. 2018; 51(2):e12434. doi:10.1111/cpr.12434

18. Kassem M, Kristiansen M, Abdallah BM. Mesenchymal stem cells: cell biology and potential use in therapy. Basic Clin Pharmacol Toxicol. 2004;95(5):209-214.

19. Abbaszadeh HA, Tiraihi T, Delshad A, et al. Differentiation of neurosphere-derived rat neural stem cells into oligodendrocyte-like cells by repressing PDGF- $\alpha$ and Olig2 with triiodothyronine. Tissue Cell. 2014;46(6):462-469. doi:10.1016/j.tice.2014.08.003

20. Darabi S, Tiraihi T, Delshad A, Sadeghizadeh M, Khalil $\mathrm{W}$, Taheri T. In vitro non-viral murine pro-neurotrophin 3 gene transfer into rat bone marrow stromal cells. J Neurol Sci. 2017;375:137-145.
21. Shams Nooraei M, Noori-Zadeh A, Darabi S, Rajaei F, Golmohammadi Z, Abbaszadeh HA. Low level of autophagy-related gene 10 (ATG10) expression in the 6-hydroxydopamine rat model of Parkinson's disease. Iran Biomed J. 2018;22(1):15-21. doi:10.22034/ibj.22.1.15

22. Noorafshan A, Abdollahifar MA, Asadi-Golshan R, Rashidian-Rashidabadi A, Karbalay-Doust S. Curcumin and sertraline prevent the reduction of the number of neurons and glial cells and the volume of rats' medial prefrontal cortex induced by stress. Acta Neurobiol Exp (Wars). 2014;74(1):44-53.

23. Nakano N, Nakai Y, Seo T-B, et al. Characterization of conditioned medium of cultured bone marrow stromal cells. Neurosci Lett. 2010;483(1):57-61.doi:10.1016/j. neulet.2010.07.062

24. Linero I, Chaparro O. Paracrine effect of mesenchymal stem cells derived from human adipose tissue in bone regeneration. PLoS One. 2014;9(9):e107001.doi:10.1371/ journal.pone.0107001

25. Hao P, Liang Z, Piao H, et al. Conditioned medium of human adipose-derived mesenchymal stem cells mediates protection in neurons following glutamate excitotoxicity by regulating energy metabolism and GAP-43 expression. Metab Brain Dis. 2014;29(1):193-205. doi:10.1007/s11011014-9490-y

26. Horie $\mathrm{M}$, Choi $\mathrm{H}$, Lee $\mathrm{RH}$. Intra-articular injection of human mesenchymal stem cells (MSCs) promote rat meniscal regeneration by being activated to express Indian hedgehog that enhances expression of type II collagen. Osteoarthritis Cartilage. 2012;20(10):1197-1207. doi:10.1016/j.joca.2012.06.002

27. de Almeida PE, Ransohoff JD, Nahid A, Wu JC. Immunogenicity of pluripotent stem cells and their derivatives. Circ Res. 2013;112(3):549-61.doi:10.1161/ CIRCRESAHA.111.249243

28. Garavello I, Baranauskas V, da Cruz-Hofling MA. The effects of low laser irradiation on angiogenesis in injured rat tibiae. Histol Histopathol. 2004; 19(1):43-48. doi:10.14670/ $\mathrm{HH}-19.43$

29. Sanchooli T, Norouzian M, Ardeshiry lajimi A, et al. Adipose Derived stem cells conditioned media in combination with bioceramic-collagen scaffolds improved calvarial bone healing in hypothyroid rats. Iran Red Crescent Med J. 2017;19(5); e45516.

30. Kawai T, Katagiri W, Osugi M, Sugimura Y, Hibi H, Ueda M. Secretomes from bone marrow-derived mesenchymal stromal cells enhance periodontal tissue regeneration. Cytotherapy. 2015;17(4):369-381. doi:10.1016/j. jcyt.2014.11.009 